\title{
Patients with diabetic eye disease using a potentially therapeutic mask. Do sufficient patients wear the mask and for how long?
}

\begin{abstract}
A single centre, prospective, interventional, open label clinical trial was conducted at Vinohrady Eye Department, Prague to evaluate safety and the mask wearing characteristics of patient volunteers who agreed to night-time use of the Noctura 400 light mask for treatment of diabetic retinopathy (DR). Noctura 400 shines green light through closed lids to prevent dark adaption and elevated retinal oxygen consumption while the patient sleeps. A total of 45 patients with advanced non-proliferative or early proliferative DR were recruited and all had diabetic macular oedema (DME). The patients wore the Noctura 400 mask for up to 8 hours per night for six months with measurements of Visual Acuity and Macular thickness at three monthly intervals. The Noctura 400 mask recorded their nightly use. Of the 45 patients who began the trial, 35 (78\%) completed the 6 months of nightly mask wear and no major safety issues were identified. Electronic monitoring showed that patients wore the mask for $4.96+/-2.97$ hours per night and there was no significant lack of persistence over the 6 months trial duration. The average number of missed nights of wear per patient was $34.1+/-39.0$. Visual acuity in these patients showed a modest improvement reversing the visual deterioration noted over 10 months prior to recruitment. Also there was central macular thinning in $38.8 \%$ of the completing patients. This commercial device appears to be safe, is worn by the majority of patients with some evidence of benefit even in advanced cases of DR.
\end{abstract}

Keywords: diabetic retinopathy, phototherapy, hypoxia, sleep mask, dark adaptation, retina, retinopathy, noctura
Volume 7 Issue 7 - 2017

\author{
Miroslav Veith,' Pavel Kuchynka,' lan \\ Grierson, ${ }^{2}$ Duncan Hill ${ }^{3}$ \\ 'Vinohrady Teaching Hospital, Czech Republic \\ ${ }^{2}$ St Paul Eye Unit and the Department of Eye and Vision Science, \\ UK \\ ${ }^{3}$ PolyPhotonix Ltd, UK
}

\begin{abstract}
Correspondence: Miroslav Veith,Vinohrady Teaching Hospital, Ophthalmology Department, Srobarova II I0- I I 50, 100 34, Prague 10 Czech Republic, Email mveith@email.cz
\end{abstract}

Received: December 0I, 2017 | Published: December I8,
2017
Abbreviations: DR, diabetic retinopathy; DME, diabetic macular edema; OCT, optical coherence tomography; BCVA, best corrected visual acuity; OLED, organic led; CST, central subfield thickness

\section{Introduction}

The retina has one of the highest oxygen consumptions of any tissue in the body, ${ }^{1}$ and this is mainly due to the photoreceptors and the rods in particular. ${ }^{2}$ The rod consumption of oxygen is at its greatest when the retina is dark-adapted, ${ }^{3}$ and the rods generate dark current. At such time the choroid, the main vascular supply to the photoreceptors, is at its most sluggish and so greater demand is needed from the retinal circulation of the inner retina. ${ }^{4,5}$ In diabetic retinopathy, the inner retinal circulation is compromised so the enhanced oxygen requirement of the dark-adapted rods is likely to be a major vascular stress inducer, contributing to the retinal pathology and compromised retinal tissue. ${ }^{6}$

In the modern world full dark adaption rarely takes place except during sleep. ${ }^{4}$ Thus prevention of rod dark-adaption by use of a nighttime light mask is a potentially important home-based, therapeutic option for diabetics with retinopathy and diabetic macular edema (DME). The particular nightly duration of mask wear depends on the patient's sleep, with the goal being to suppress dark adaptation using the mask for as much time that the patient sleeps as possible. It was shown in a UK safety trial (INSIGHT trial) that both young and elderly normal volunteers and a limited number of patients with diabetic retinopathy wore a light mask nightly for 3 months without major safety issues, ${ }^{7,8}$ including no significant concerns regarding disruption of circadian rhythms or other sleep issues due to the light. In addition, a proof of concept trial also in the UK, has shown that diabetics with retinopathy wore another type of light mask successfully for 6 months without adverse events. ${ }^{9}$ Although this trial (conducted at Kings Hospital, London) was not powered for efficacy, nonetheless it was encouraging that at the outset of the investigation 28 out of 34 trial-completing retinopathy patients had macular cysts and this was reduced to 19 at trial end. ${ }^{9,10}$ Similarly INSIGHT's 15 retinopathy patients had 12 with macular cysts and, of these 10, patients exhibited reduction or elimination of cysts following 3 months of mask wear. ${ }^{8}$

For the KINGS trial, ${ }^{9}$ the light mask contained light emitting diodes (LEDs) that could be recharged from the mains as required. On the other hand, the INSIGHT trial ${ }^{7,8}$ used a commercially constructed mask incorporating a pod with Organic LEDs (OLEDs). An OLED is carbon-based technology ${ }^{11}$ providing a uniform, glowing illumination for both eyes. Importantly the pod also contains mask wear monitoring so that a precise record of mask use was available for each volunteer. Objective monitoring of non-ocular external devices often show poor compliance among diabetics. ${ }^{12}$ It is known from electronic monitoring in other areas of ocular interest that for instance inadequate wearing of occlusive patches ${ }^{13}$ contributes in no small way to mediocre visual outcomes. In other words it cannot be assumed in any way that adequate home provision of the device will equate to appropriate use. Indeed adherence to recommended treatment irrespective of patient age or nature of illness can be very disappointing. ${ }^{14}$ It follows that no 
matter how potentially therapeutic the mask might turn out to be, it is of little consequence if it is not worn sufficiently and for long enough. It is intended that these concerns be addressed in the present study.

The light mask has subsequently been called Noctura 400 (the device was CE marked and manufactured and certified based on ISO13485) and it was used in the present prospective, open-label, clinical trial involving patients with moderate proliferative or nonproliferative diabetic retinopathy (DR) and progressive DME. The aim was to determine with as much accuracy as possible how regularly and frequently patients wore the mask, if persistency was an issue after several months of wear and in a patient population whether adverse events were associated with light mask use. A total of 45 patients were recruited in the Czech Republic for the additional safety trial but this time all recruits had DR and the volunteers were required to wear the Noctura 400 light mask for 6 months. The present study allowed us to ask the additional question "did the DR subjects wear Noctura 400 and if so how often and for how long?" Safety and wellbeing questions were addressed plus anatomic (spectral domain optical coherence tomography (OCT) of macular thickness) and functional (best corrected visual acuity, BCVA) indicators of disease status were measured.

\section{Materials and methods}

The investigation was conducted at Vinohrady Teaching Hospital Ophthalmology Department, Prague, Czech Republic. It was a single centre, prospective, interventional, open label clinical trial evaluating, among other objectives, the mask wearing characteristics of patient volunteers who agreed to night-time use of the Noctura 400 light mask. The study conformed to the World Medical Association declaration of Helsinki on "Ethical Principles for Medical Research Involving Human Subjects". Approval was obtained from the Vinohrady Hospital Ethics Committee and the trial was conducted under the regulations of the Czech Republic (Act No 123/2000 Coll, Decree No 316/2000 Coll and Government Regulation No 336/2004 Coll and No 163/2002 Coll). Trial registration details can be found at http://www.isrctn.com/ISRCTN81658067.

\section{Recruitment and eligibility}

All recruited diabetic volunteers provided informed, written consent after full explanation of the nature of the mask, comprehensive guidance from a trial member on how to use the mask at bed time, possible contraindications, the objectives of the trial and what they were required to do for the 6 months of trial duration. The volunteers were patients with DR and each received from the outset an information package and a patient diary. In the diary the patients were requested to record their daily sleep patterns, disturbances if any and the degree of comfort of the mask.

Patients included had central macular thickness, based on spectral domain optical coherence tomography (OCT) measurement, of 220 $\mu \mathrm{m}$ or greater in either eye. Exclusion criteria included any ocular or systemic condition, other than DR and type I or tHbype II diabetes that affected the blood-retinal barrier. A history of sleep disturbances or depression and the regular use of sleeping tablets also warranted exclusion from the trial. Duration of DME was not an exclusion criterion nor was early macular tractional changes. In addition control of diabetes based on measurement of glycated haemoglobin $\left(\mathrm{HbA}_{1 \mathrm{c}}\right)$ was not an exclusion criterion but these were measured at baseline and after 6 months. Other systemic criterion such as systemic blood pressure and total cholesterol were taken at baseline and study end.

\section{Mask intervention}

The light mask used for this clinical trial, Noctura 400, was developed by PolyPhotonix Medical Ltd, UK and distributed in the Czech Republic by Elon Medical s.r.o.. The mask consists of two parts; a plastic pod and a fabric mask into which the pod is housed when in use (Figure 1). The pod incorporates two OLED light sources, which have a peak light emission of $505 \mathrm{~nm}$ close to the wavelength of maximum rod sensitivity and powered by integral batteries. The light intensity was set at $74+/-10 \mathrm{~cd} / \mathrm{m}^{2}$ and is similar to that used in light therapeutic devices for treatment of DR patients by others. ${ }^{10,15}$ Light of such predominantly green emission and appropriate intensity was chosen because after passing through closed lids as the user sleeps it has been estimated to be sufficient at the retina to effectively suppress dark adaption..$^{16,17}$

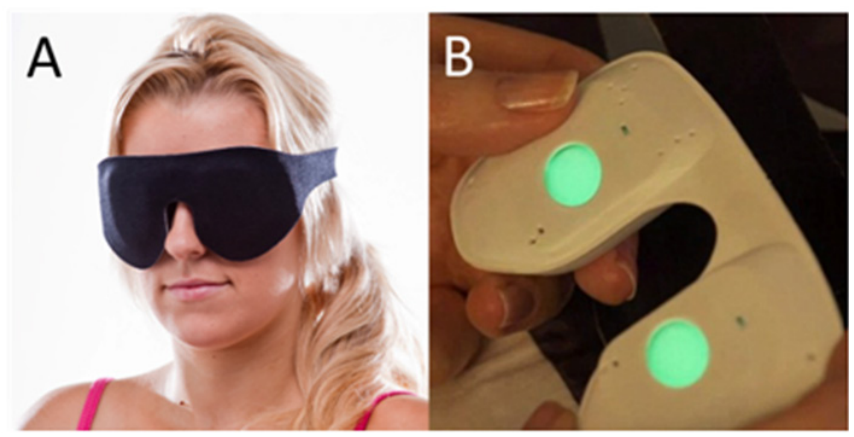

Figure I (A) The patient wears the Noctura mask when they go to bed. The mask houses (B) a pod that is activated by touch and then slotted into place in the mask. The gently glowing green OLED lights glow into the eyes through closed lids.

Patients were asked to wear the mask nightly for 6 months and each patient was given a new mask and pod monthly with a telephone call backup to each participating patient. When going to sleep the patient activates the Noctura 400 light source in the pod (Figure 1B) and then locates the activated pod within the mask housing. The mask can then be fitted comfortably over the eyes and fastened in place with Velcro straps (Figure 1A). The patient is initially aware of a gently glowing green light through closed lids. As OLED emission is homogenous and isotropic, eye movement during sleep does not effectively alter the degree of retinal illumination. Patients adapt to this constant light exposure through the Troxler Effect, ${ }^{18}$ and no longer are aware of the green lighting after a few minutes.

The masks are designed to work within an operational window that can be programmed to encompass the patient's routine sleep pattern, typically from 20:00-08:00 each night. During this operational window capacitive sensors housed in the pod and lying just under the eyes during sleep require close facial proximity for the OLEDs to operate. Integrated firmware detects every second if the mask is in operational use or not and stores this binary information at two minute intervals in an on-board memory chip providing an effective and accurate mechanism to record the mask wearing habits of each of the trial patients. The maximum period of illumination is programmable and was set arbitrarily at 8 hours for this present study.

\section{Outcome measures}

The used masks were examined for wear and tear each month when they were returned and at the same time the pods were sent to the reading centre (PolyPhotonix Medical, Sedgefield, UK) for monthly determination of mask usage. The mask utilisation data for 
each coded patient was read using near field communication (NFC) chip (M24LR64A chip) and the data was downloaded wirelessly, using radio-frequency identification (RFID) technology. A unique identifier on each masks served to link each pod to the appropriate patient ensuring the accurate matching of the pod data and patient whilst maintaining anonymity. The data consists of "on" and "off" information at two-minute intervals throughout the operational period and the data block is interpreted using a CR95HF RF transceiver (ST Microelectronics). A presentational histogram of such data can be seen in Figure 2. The 32 columns of the graph contain the maximum possible number of daily operational windows for one month's mask use. The green and red components of each column indicate when the mask is in actual operational use as a green line whereas if either it is not being worn or just not lit the column is red during that time period (Figure 2)

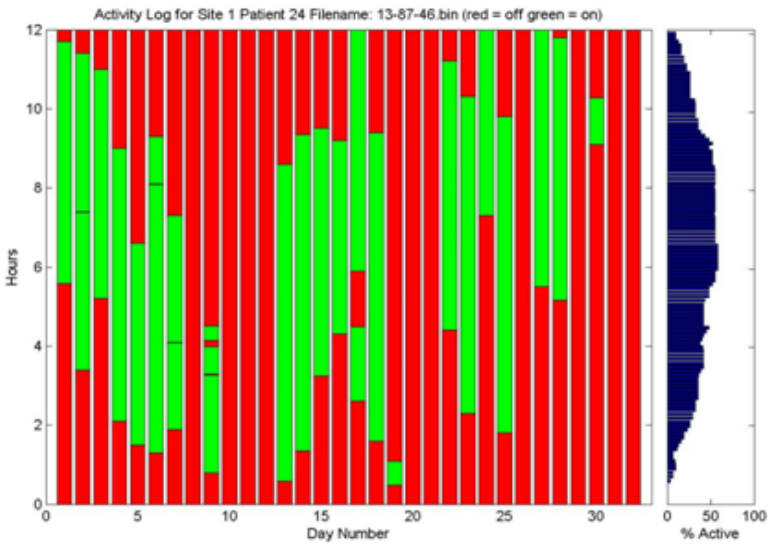

Figure 2 One single month of recording of mask wear to explain the different elements in the recording. The green lines are the nightly periods of mask wear and this patient No24 accumulated I 34.15 hours of wear in that month averaging 4.33 hours per night. The patient, in common with many diabetics, appears to have an erratic bedtime schedule. Truncated night time mask wear is seen on days 9,19 and 30. Also of concern with this patient, however, is the failure to have any mask wear on eight days including on two occasions there are sequences of three days (days I0-12) and two days (days 20 and 2I) mask free.

Clinical outcome measures were assessed at baseline, at $3+$ months (half way point) and at 6 months. Medical and ocular history was taken as part of a routine ophthalmic examination. Patients were monitored for treatment-related side effects and complications. Adverse events were recorded, assessed for severity and attribution and reported in line with European Directive 2001/20/EC. At each visit the patient returned their diary and an informal discussion on mask wear issues was undertaken with a health care professional. At the exit visit the patients were asked to fill out a questionnaire that helped direct the safety and tolerance discussion. BCVA was assessed at 4months using Early Treatment Diabetic Retinopathy (ETDRS) boards. In addition retrospective collection of pre-study BCVA was collated from clinical records. The average time these were recorded before the study baseline was 10 months. Spectral domain optical coherence tomography (SD-OCT) (Heidelberg Engineering) was used to determine macular thickness and changes to the vitreoretinal interface. A retinal thickness map centred on the fovea and measurements made of central subfield thickness (CST), cube volume (CV) and cube average thickness (CAT).

\section{Analysis}

Mask wear data from the applicable patients was collated allowing us to calculate the mean mask wear per individual patient for the whole length of study, each month and also each night of the investigation across the length of the study. Further, the number of nights where there was no mask wear at all was noted for each patient. In addition to mask usage the trial explored the impact of the eye mask upon the progress of ocular disease and function. A statistical significance at the $5 \%$ level in any of the measures explored was a used as a marker to inform further future research.

\section{Results and discussion}

The trial enrolled 45 patients with a mean age of 62.5 years (range 31 to 79 years). Of these 8 had type I diabetes established for on average 29.8 years and 37 had type II diabetes with an average duration of 16.5 years (see Table 1 showing patient characteristics). They were predominantly males $(73 \%)$ with currently diffuse $(84 \%)$ rather than focal (16\%) DME that had first been diagnosed on average 2.4 years prior to the start of the trial. Many had macular laser in the past (over 70\%) and some had intravitreal injections but these were in the minority. Time since last intervention averaged 1 year for left eyes and 8 months for right eyes see Table 1 .

Table I Patient characteristics (numeric variables, parametric summary) giving totals (All) and those that completed the study (Evaluable)

\begin{tabular}{|c|c|c|c|c|c|c|}
\hline & \multicolumn{3}{|l|}{ All } & \multicolumn{3}{|c|}{ Evaluable } \\
\hline & Mean & (SD) & $\mathbf{N}$ & Mean & (SD) & $\mathbf{N}$ \\
\hline Age, $y$ & 63.0 & $(10.2)$ & 45 & 62.6 & $(9.7)$ & 35 \\
\hline Type I Diabetes & 49.5 & $(12.7)$ & 8 & 51.6 & $(12.1)$ & 7 \\
\hline Type II Diabetes & 65.9 & $(6.8)$ & 37 & 65.3 & $(7.0)$ & 28 \\
\hline $\mathrm{HbA}_{\mathrm{Ic}}$ & 67.9 & $(15.1)$ & 44 & 66.2 & $(15.4)$ & 35 \\
\hline BP Systolic, $\mathrm{mmHg}$ & 132.5 & $(15.0)$ & 44 & 135.1 & $(15.3)$ & 35 \\
\hline BP Diastolic, mmHg & 79.4 & $(8.7)$ & 44 & 79.6 & $(9.2)$ & 35 \\
\hline Visual acuity, \% (L) & 0.46 & $(0.30)$ & 44 & 0.48 & $(0.30)$ & 34 \\
\hline Visual acuity, \% (R) & 0.40 & $(0.26)$ & 45 & 0.39 & $(0.27)$ & 35 \\
\hline OCT (CST), $\mu \mathrm{m}$ (L) & 386.9 & $(168.3)$ & 40 & 393.0 & $(\mid 77.6)$ & 34 \\
\hline OCT (CST), $\mu \mathrm{m}(\mathrm{R})$ & 378.1 & $(142.3)$ & 41 & 375.1 & $(149.5)$ & 35 \\
\hline
\end{tabular}


Table Continued..

\begin{tabular}{|c|c|c|c|c|c|c|}
\hline & \multicolumn{3}{|l|}{ All } & \multicolumn{3}{|c|}{ Evaluable } \\
\hline & Mean & (SD) & $\mathbf{N}$ & Mean & (SD) & $\mathbf{N}$ \\
\hline Time with diabetes diagnosed, $y$ & 18.9 & $(11.3)$ & 44 & 18.9 & $(11.5)$ & 35 \\
\hline Type I Diabetes & 29.8 & $(12.1)$ & 8 & 31.2 & $(12.4)$ & 7 \\
\hline Type II Diabetes & 16.5 & $(9.7)$ & 36 & 15.9 & $(9.1)$ & 28 \\
\hline Time since DME diagnosed, y $(L)$ & 2.4 & $(2.1)$ & 44 & 2.4 & $(2.0)$ & 34 \\
\hline Time since DME diagnosed, y (R) & 2.4 & $(2.1)$ & 44 & 2.6 & $(2.2)$ & 35 \\
\hline Time since laser therapy, $y(L)$ & 1.8 & $(1.6)$ & 31 & 2.0 & $(1.7)$ & 26 \\
\hline Time since laser therapy, $y(R)$ & 1.9 & $(1.9)$ & 34 & 2.1 & $(2.0)$ & 26 \\
\hline Time since IV, y (L) injection, y (L) & 1.0 & $(0.9)$ & 7 & 1.1 & $(0.9)$ & 6 \\
\hline Time since IV, y (L) injection, y (R) & 0.7 & $(0.6)$ & 17 & 0.7 & $(0.6)$ & 13 \\
\hline
\end{tabular}

\section{Primary outcomes - safety and mask use}

A total of 35 patients completed the trial (78\%) and of the 10 who failed to complete it is known definitely that only $3(7 \%)$ were for mask related events of either light intolerance or mask wear intolerance. A further 3 suffered non-mask related severe adverse events (one death, one developed ischemic heart disease and the third developed severe diabetes-related foot complications). Of the remaining 4, the reason for trial exit was unknown. Noteworthy was that all patients who reached the 3 month clinical assessment point went on to complete the trial (Figure 3).

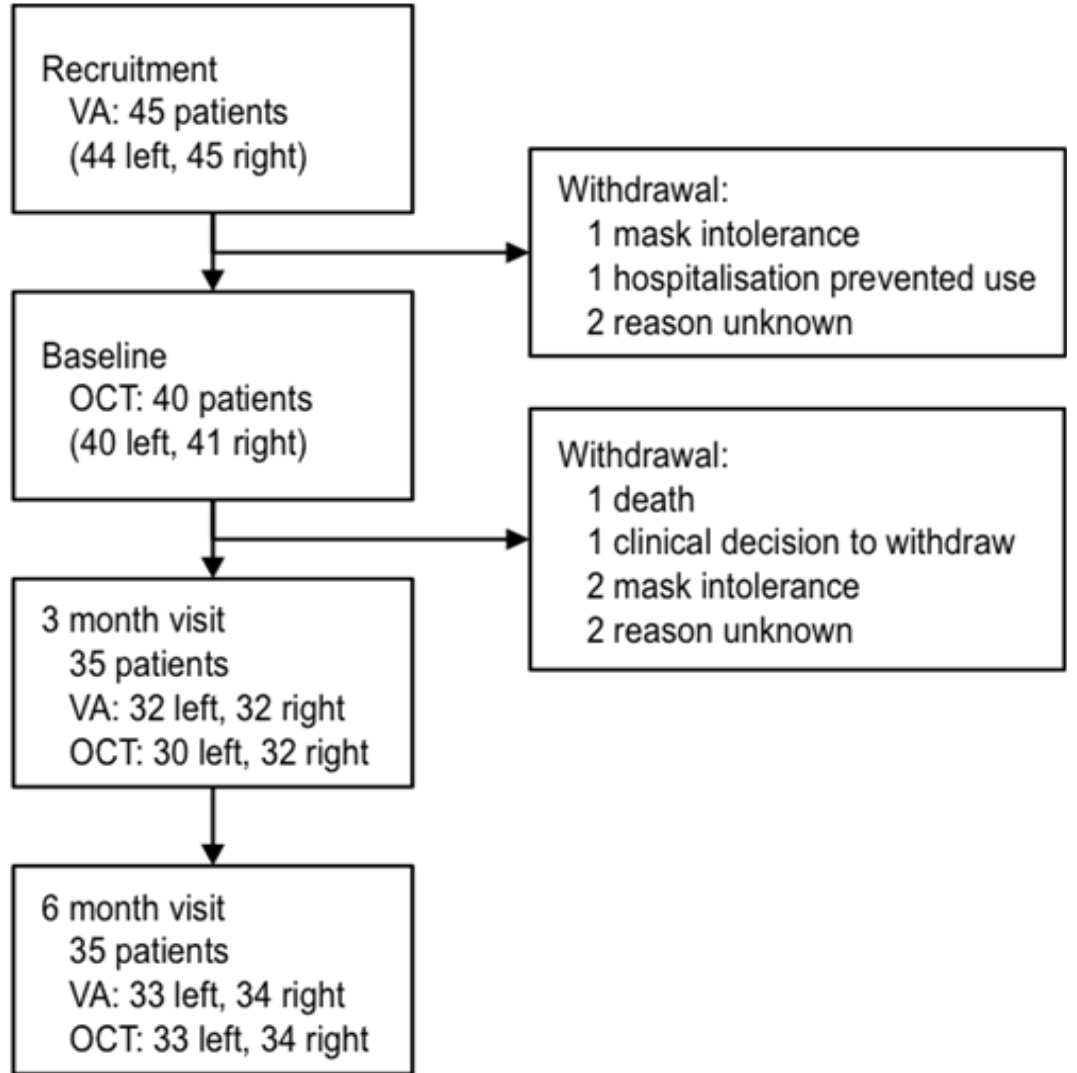

Figure 3 Flow diagram showing in the sequence boxes trial recruitment, follow up examination by VA and OCT and withdrawals from the study. 
No major safety issues were identified in this study related to mask wear. However the sleep diary examination identified mask discomfort, rubbing of straps and other design related features as issues for a minority of patients. The patient questionnaire suggested that $23 \%$ found the mask uncomfortable (Table 2). In conjunction a small proportion $(14 \%)$ of those who completed the trial reported sleeping difficulties during the 6 months trial that were beyond what they had noted prior to mask wear. In addition, only 1 person would not recommend the mask to others but this has to be tempered by the fact that at the end of the 6 months, $26 \%$ felt that they themselves were not willing to continue mask wear were the situation to arise (Table 2).

Table 2 Patient questionnaire; questions and answers

\begin{tabular}{lllllll}
\hline & No & \multicolumn{3}{l}{ Yes } & \multicolumn{2}{c}{ Unsure } \\
\hline Q1 New sleeping difficulties? & 29 & $83 \%$ & 5 & $14 \%$ & I & $3 \%$ \\
Q2 New trouble falling asleep? & 31 & $89 \%$ & 3 & $9 \%$ & I & $3 \%$ \\
Q3 Mask light intrusive? & 29 & $83 \%$ & 5 & $14 \%$ & I & $3 \%$ \\
Q4 Uncomfortable to wear? & 27 & $77 \%$ & 8 & $23 \%$ & & \\
Q5 Willing to continue using? & 9 & $26 \%$ & 18 & $51 \%$ & 8 & $23 \%$ \\
Q6 Recommend using? & I & $3 \%$ & 22 & $63 \%$ & 12 & $34 \%$ \\
& Very Satisfied & Satisfied & Neither S or D \\
Q7 Overall satisfaction rating? & 3 & $9 \%$ & 23 & $66 \%$ & 9 & $26 \%$ \\
\hline
\end{tabular}

QI Have you experienced any new sleeping difficulties while using the mask?

Q2 Have you experienced newly formed trouble falling asleep while using the mask?

Q3 Did you find the mask light intrusive?

Q4 Was it uncomfortable to wear the mask otherwise?

Q5 Would you be willing to continue to use the mask?

Q6 Would you recommend the mask to other DR patients?

Q7 How would you rate the overall light therapy mask treatment?

The mask wear monitoring used in this study was found to be a very effective way of precisely determining the mask wearing habits of the patients. Mask wearing history can be seen in the example shown where the whole 6 month profile of one patient is outlined graphically (Figure 4). Although the patient has worn the mask most nights there are three types of lapses that can be identified in the mask wear history. The first is an occasional lost night here and there and the second is probable lost hours in a specific night as indicated by a truncated night-time period of mask to face contact (Figure 4). A failure to wear the mask over a series of sequential days or weeks is a third type of lapse (Figure 2).

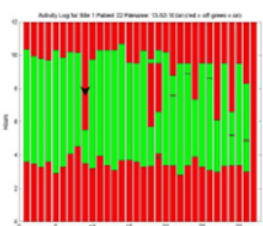

a. March

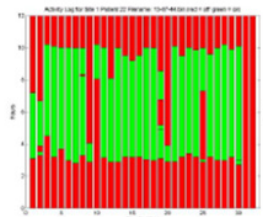

c. May

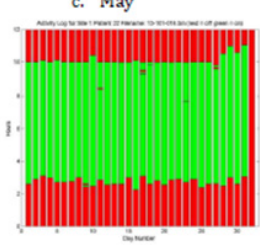

e. July

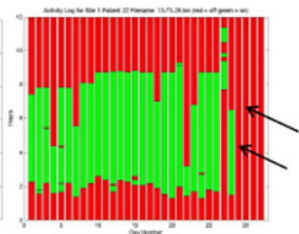

b. April

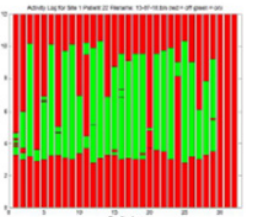

d. June

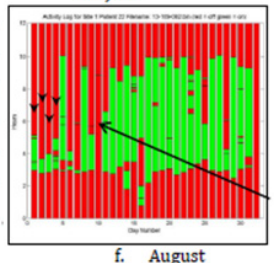

Figure 4 Graphs a to f show the 6 months from March to August respectively of mask use for one patient throughout the trial. The bars in each graph denote the daily operational windows of each month's mask use. The green indicates when the mask is in operational use and the red when the mask is not in use for each day. The vertical axis shows the time in hours from the commencement of the operational window, 8pm - 8am (local time). Arrows show whole nights missed such as 29th and 30th April and 10th August. Arrow heads show examples of interrupted (truncated) periods of mask to face contact such as the first one on 9th of March and a series from the Ist to 4th of August. Note that July is a consistent and almost perfect month of mask wear for this patient. 
It can be seen from the monitoring data that most people in the study wore the mask at night-time (Figure 5) but the mask wear fell short of the maximum 8 hours available as was expected for an elderly population of unwell patients. For this study the patients wore the mask for 4.96+/-2.97 hours per night on average over the 6 months although there was much variation between individuals (Figure 5). Only 2 of the 35 completing patients fell below 2 hours of mask wear per night. The study accumulated 30,408 hours of mask wear averaging out at over 5000 hours per month for the 35 participants who completed the study.

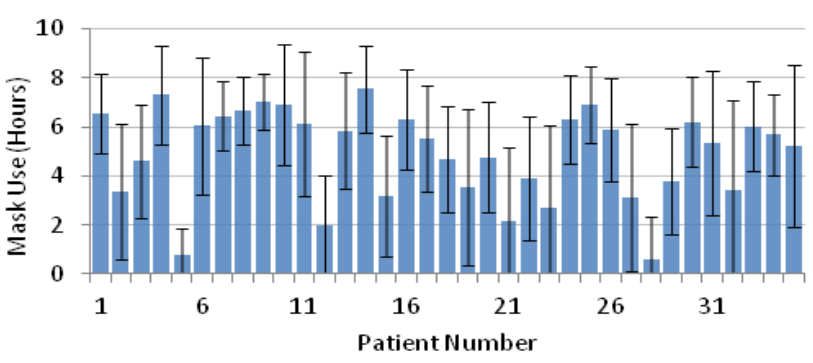

Figure 5 Histogram showing mean nightly mask use (hours) and standard deviations for each completing patient over the full six months of the study. The large variability in usage between patients is evident.

There was also some variation between months that started after the first month with a slight step up over the subsequent three months that tailed off with some evidence of minor deterioration in mask wear thereafter. The downward trend in mask wear was slight and did not reach significance indicating an absence of any marked lack of persistence (Figure 6). Diary entries help explain periods of poorer mask wear for example July and August 2013 (5th and 6th month of records) were hot summer months in Prague and night time discomfort may in part (along with holidays and the like) account for the slight drop off in mask wear (Figure 6). Arbitrarily the patients were divided into good night time mask use (over 200 hours mask wear per month), reasonable mask use (between 100 and 200 hours per month) and questionable poor use of the mask (under 100 hours per month). At the end of the first month, $82.9 \%$ belonged to the first two groups, and the trial finished with $77.1 \%$ in those two groups at completion of the sixth month with the third month having the highest incidence of $88.6 \%$.

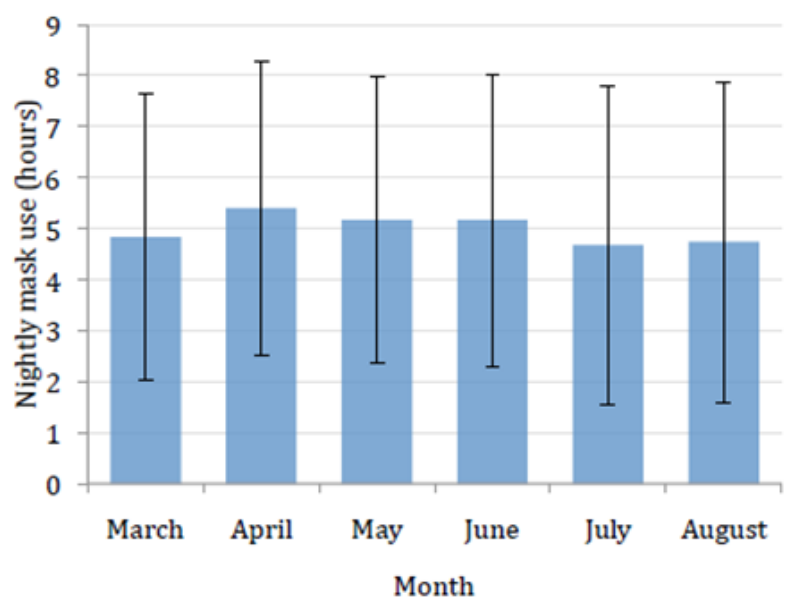

Figure 6 Graph showing mean with standard deviation of nightly mask use (in hours) for the 35 patients who completed the study in each of the months of the study duration.
Nights with limited or truncated periods of mask wear were probable but not definite indicators of failure to prevent an episode of dark adaption. On the other hand, single or multiple nights of complete absence of mask wear were strong indicators of noncompliance. Analysis of mask wear electronic monitoring profiles for each completing patient showed lost nights amounted to an average $34.1+/-39.0(18.5 \%)$ of the total of 184 trial nights. None of the 35 completing patients was without lost nights but $17(48.6 \%)$ had trivial (less than 14) lost nights during the study. At the other extreme 5 $(14.3 \%)$ completed the trial but they were shown to wear the mask for less than half of the total available nights. On the basis of nights lost to treatment $85.7 \%$ of the completing patients were within what is being considered 'the zone of potential therapy' while the rest had more limited restriction of their dark adaption.

\section{Secondary outcomes - BCVA and OCT}

The functional analysis showed that mean glycated haemoglobin levels(HBA1c) during the test period for the patients were $67.9+/$ $15.1 \mathrm{mmol} / \mathrm{mol}$ at 0 months (trial start) and $66.2 \mathrm{mmol} / \mathrm{mol}$ at 6 months (trial end) $(p=0.12)$. Contrary to the expectation of progressive disease-associated decline in VA, it improved marginally during the 6 months of follow-up from a mean and SD LogMAR of $0.45+/-0.37$ to $0.39+/-0.33$ left eye and $0.53+/-0.35$ to $0.46+/-0.34$ right eye. Records of pre-study BCVA were available for on average 10 months (range 1 to 54 months) prior to trial baseline for the study patients. These were assessed to determine whether the direction of BCVA was similar or otherwise to that trend shown during the study period. Prestudy BCVA declined to baseline (pre study LogMAR means were $0.41+/-0.30$ left eye and $0.49+/-0.36$ right eye) such that on average there was an approximate $9 \%$ deterioration in vision that became a 13 to $14 \%$ improvement over the 6 months of mask wear so more than reversing the downward trend.

The OCT measurements of Central Subfield Thickness (CST) showed that there was clear macular thinning in the eyes of some patients over the 6 month period however there was a number who stayed the same and others who got worse. When a cut of $15 \mu \mathrm{m}$ either way was taken as being noise, of the total of 67 eyes from the 35 completed patients, then $26(38.8 \%)$ improved, 20 (29.9\%) remained the same and $21(31.1 \%)$ deteriorated. Central Subfield Thickness (CST) at the start of the trial was $393+/-178 \mu \mathrm{m}$ (left eye) and 375+/$150 \mu \mathrm{m}$ (right eye) and fell to $381+/-156 \mu \mathrm{m}$ (left eye) and $348+/-127$ $\mu \mathrm{m}$ (right eye) at trial end; a thinning of $12 \mu \mathrm{m}$ and $27 \mu \mathrm{m}$ respectively.

A therapeutic sleep mask to treat DME and other pathologies associated with diabetic eye disease by preventing dark adaption and reducing night-time oxygen consumption may be an important new development by providing an inexpensive home treatment for a growing worldwide ophthalmic problem. ${ }^{19}$ The therapeutic potential of Noctura 400 and similar masks, ${ }^{7,9}$ is encouraging, but no Phase III efficacy trials have been published for such devices at the time of writing. No matter how potentially effective the sleep mask might be, it will be of little use if patients find it unsuitable for regular use.

It has been shown in predominantly normal volunteers plus some with diabetic eye disease (INSIGHT) ${ }^{7,8}$ that wearing Noctura 400 on a regular basis is safe and this earlier finding has been supported by the present study confined exclusively to patients with diabetic eye disease. It was reassuring in this investigation to find that $78 \%$ of the trial subjects completed the 6 month study so routine mask wear is a practical proposition for most potential patients. The $78 \%$ figure is well in line with the extensive general medical "adherence 
to treatment" meta analysis of 569 studies which calculated a $75.2 \%$ overall average, ${ }^{20}$ of the patients that dropped out, only $7.0 \%$ of "the lost from trial" were definitely mask wear or light exposure related. Where those for which the exit reason was unknown are added in or left out, then the range became $15.6 \%$ at most to $7.0 \%$ at the lowest. The findings contrast somewhat with the shorter INSIGHT trial where $27 \%$ were lost because of mask/light related issues. ${ }^{7}$

It was not the case that the $78 \%$ of patients who completed the trial were entirely problem free because a minority $(23 \%)$ reported mask discomfort and a lower number (14\%) had some minor sleeping difficulties. These factors may have reduced overall mask wear and suggest further comfort-related design adjustments to the mask which was also suggested in the report of the INSIGHT study run on predominantly normal volunteers. ${ }^{8}$ That there was more mask issues and more drop outs in INSIGHT raises the likelihood that the patients in this present trial were more motivated to mask wear than healthy individuals.

Clearly, even although there are other variables to consider, it seems likely the target diabetic eye disease population persisted with the daily therapy somewhat better than normals but both studies showcased the benefits of electronic monitoring. The accuracy of electronic monitoring over other forms of therapy adherence evaluation is well established in the literature, ${ }^{21}$ indeed both eye drop ${ }^{22}$ and ocular device ${ }^{23}$ electronic monitoring have been around for some time. It is over 20 years ago that Fielder and co-workers highlighted the value of electronic monitoring of patching in paediatric ophthalmology. The present trial provided us with more than 30,000 hours of Noctura 400 patient wear and confirmed to us that the mask utilisation monitor in the pod was a powerful tool within this potential new therapeutic direction. This allows us in "real world" medicine to monitor the sleep mask usage of each of the patients and if ever mask wear is poor to council patients positively or alternatively if the mask is ineffective to alter therapeutic options.

It was encouraging that most patients were able to wear the mask and the monitoring device told us that mask wear averaged out at around 5 hours per night that was in line with the slightly greater than 5 hours per night for the DME subjects from INSIGHT (calculated from their quoted $495.8 \mathrm{hrs}$ over 3 months per patient ${ }^{7}$ The question.... arises whether longer mask wear than this could be expected. The mask discomfort issues have already been discussed and undoubtedly each patient had lost nightly therapy when they either missed out a night completely or only wore the mask for part of their sleep cycle. In addition there is a slight drop in mask wear in the last three compared to the first three months. The drop off may be just coincidental as it was not particularly large however the other two possibilities were 1) a level of mask fatigue or 2) higher night-time temperature of the summer months making the mask less comfortable to wear.

Clearly the patients fell short of the maximum OLED lighting exposure of 8 hours programmed into the pod averaging $60 \%$ of that time available. On the other hand the 8 hours was always an arbitrary choice of time that provided a maximum possible exposure while preserving battery life over each month. There is no precise figure for the actual sleep time per night for each patient only when and how long they wore the mask. As such the average mask wear per patient is a somewhat flawed surrogate for true compliance. Indeed exit interview and diary entries tell us that most if not all patients had periods of sleep without mask activation. However the clearest indicator of lost periods of potential therapy is the single or multiple nights of complete absence of mask wear. All 35 patients that completed 6 months had nights off but for many $(48.6 \%)$ the nights lost was trivial and the vast majority $(85.7 \%)$ wore the mask most nights. Even so an average of 34.1 nights of missed mask usage out of a possible 184 trial nights shows clear room for improvement. Nights lost is believed to be a powerful indicator of episodes of non-compliance and without doubt the main cause of most suboptimal mask wear in this clinical trial. If only nights of actual mask wear are assessed then average wear increases to 6 hours rather than 5 hours per night. Clearly most patients need to be counselled not to miss nights as these may have been through a host of reasons including choice, forgetfulness and lifestyle intrusion. The latter, especially where loss is of consecutive nights of mask wear, suggests episodes of interruption of normal routine such as holidays, hospitalisation etc.

The question arises how much suboptimal mask use might there be within those that completed the trial? To maximally compromise dark adaption in this group, who were for the most part elderly (average age over 60 years) and rather unwell, it was necessary for them to have minimal sleep without the mask. Research shows that the healthy elderly sleep only between 6-7 hours per night, ${ }^{24}$ and this becomes much closer to 6 hours when sleep is measured objectively. ${ }^{25}$ Not surprisingly sleep is even less if patients have illnesses like diabetes. For instance one large and quantitative study found that $64 \%$ of their diabetics slept less than 6 hours and 34\% were lower than 5 hours. ${ }^{26}$ In a "real world" situation (as mimicked in the present trial) the recorded sleep mask wear level of 5 hours possibly eliminates up to $70 \%$ of an average patient's nightly dark adaption if you accept an estimate of between 6 and 7 hours night-time sleep for long established diabetics as being reasonable. Hopefully, fewer nights lost to mask usage and design alteration to increase mask comfort will make further strides towards total dark adaption avoidance. It has not yet been possible, as was the case in the treatment of amblyopia study (MOTAS), ${ }^{27}$ among others, to come up with a prescribed dose requirement against which to test patient therapeutic performance. On the other hand until appropriate Phase III trials of efficacy per se and also efficacy in relation to compliance are completed and reported it will not be known how much dark adaption avoidance is needed for therapeutic optimum effect.

The current study had too few subjects to be powered for efficacy. Also the subjects consisted of such a heterogeneous group of patients, although appropriate for mask wear evaluation and safety, they were not entirely suitable for clear therapeutic assessment. It was therefore both pleasing and surprising that BCVA improved over the six months of trial duration. This difference although small is worth noting given that the pre-trial analysis of BCVA in the mask-wearing group showed a deterioration in acuity in line with the known natural history of the disease. ${ }^{28}$ Albeit a rather disparate group of patients, the altered trend from progressive vision loss to a modest reprieve is encouraging us to believe that mask-related therapeutic improvements are possible. A predictive curve indicated that just less than $1 \%$ further visual loss per month might be expected from the group of patients whereas the reality was that after 6 months of mask wear all of the previous 10 months deterioration was eliminated plus a little extra gain in VA beyond that see Figure 7. 


\section{Visual Acuity Progression}

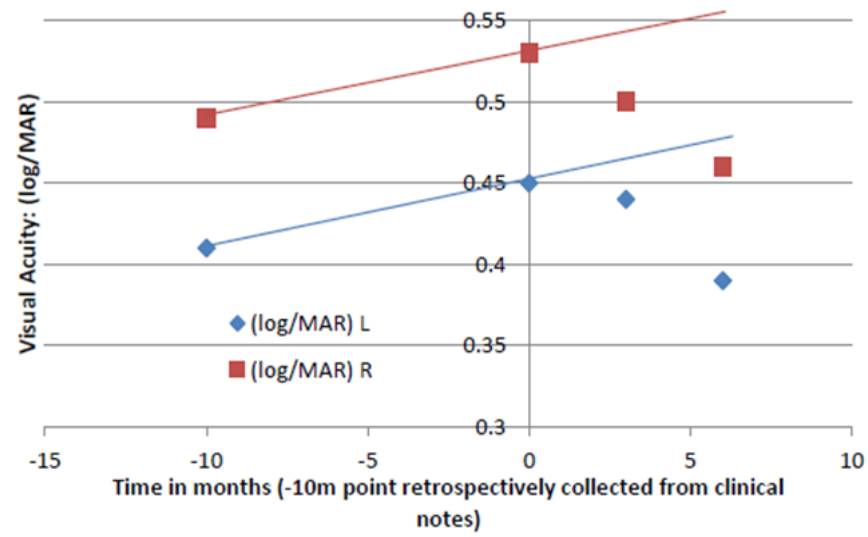

Figure $7 \mathrm{~A}$ graph showing the average LogMARVA of the completing patients over the 6 months of the clinical trial for right (red squares) and left (blue diamonds) eyes separately. In addition theirVAs prior to trial entry is included (taken on average 10 months prior to trial start point). There was a small visual decline that would reasonably have been expected to continue through the trial period (see prediction lines). However the VA in both eyes progressively improved at the 3 and 6 month time periods.

Based on OCT measurements, the CST decreased in thickness by a modest amount. However was impressive that $68.7 \%$ of the completing eyes had a CST that either improved or remained the same over the 6 month time frame. In this mask wear trial, clearly there was no parallel age and disease matched non-mask wearing control to compare anatomical improvement against. On the other hand it was a reasonable speculation that the trial group would not have been expected to fare well. The classic literature on the natural history of DME ${ }^{28}$ suggested that without intervention only $3 \%$ showed spontaneous improvement in macular swelling while $40 \%$ got worse. Further, the work of Browning \& Fraser $^{29}$ on the progress of subclinical DME (albeit followed for a year and not 6 months) showed OCT-evaluated macular thinning in a mere $10 \%$. Against these values, the $38.8 \%$ thinning and $31.3 \%$ thickening of the mask-wearing group stands up fairly well given that the patients have more established pathology. It seemed the anatomic alterations to the maculae over the 6 months of mask wear were in the right direction for many patients.

Leading on from the current mask wear trial, it is felt that nothing uncovered in this investigation (either with respect to the primary outcomes of mask adoption and safety or for that matter the secondary outcomes of BCVA and OCT) discourages the need for appropriately powered randomised control trials to provide robust evidence of Noctura 400 efficacy. However having established that a commercially-approved mask is safe and worn for extended periods by most patients, Noctura 400 has opened "a new door" for the potential treatment of those with diabetic eye disease.

\section{Acknowledgments}

This research project was partially or fully sponsored by Vinohrady Teaching Hospital and Elon Medical.

\section{Conflicts of interest} Ltd.

IG is the Medical and Scientific Adviser to PolyPhotonix Medical

\section{Funding}

None.

\section{References}

1. Schmidt M, Glessel A, Laufe T, et al. How does the eye breathe? Evidence for neuroglobin-mediated oxygen supply in the mammalian retina. J Biol Chem. 2003;278(3):1932-1935.

2. Arden GB, Sidman RL, Arap W, et al. Spare the rod and spoil the eye. $\mathrm{Br}$ J Ophthalmol. 2005;89(6):764-769.

3. Wangsa ND, Linsenmeier RA. Retinal Oxygen. Fundamental and clinical aspects. Ophthalmol. 2003;121(4):547-557.

4. Linsenmeier RA, Braun RD, McRipley MA, et.al. Retinal hypoxia in long-term diabetic cats. Invest. Ophthalmol Vis Sci. 1998;39(9):16471657.

5. De Gooyer TE, Stevenson KA, Humphries P, et al. Retinopathy is reduced during experimental diabetes in a mouse model of outer retinal degeneration. Invest. Ophthalmol Vis Sci. 2006;47(12):5561-5568.

6. Du Y, Veenstra A, Palczewski K, et al. Photoreceptor cells are major contributors to diabetes-induced oxidative stress and local inflammation in the retina. Proc Natl Acad Sci U S A. 2013;110(41):16586-16591.

7. Czanner G, Gutu T, Harding SP, et al. Safety and acceptability of an organic light emitting diode (OLED) sleep mask for the treatment of retinal diseases: INSIGHT study. Invest Ophthalmol Vis Sci. 2005;56: 161.

8. Sahni JN, Czanner G, Gutu T, et al. Safety and acceptability of an organic light emitting diode sleep mask as a potential therapy for retinal disease. Eye. 2016;31(1):97-106.

9. Arden GB, Gunduz MK, Kurtenbach A, et al. Preliminary trial to determine whether prevention of dark adaption affects the course of early diabetic retinopathy. Eye (Lond). 2010;24(7):1149-1155.

10. Arden GB, Jyothi S, Hogg CH, et al. Regression of early diabetic macular oedema is associated with prevention of dark adaption. Eye (Lond). 2011;25(12):1546-1554.

11. Geffroy B, le Roy P, Prat C. Organic light-emitting diode (OLED) technology : materials, devices and display technologies. Polymer Internat. 2006;55(6):572-582.

12. Armstrong DG, Lavery LA, Kimbriel HR, et al. Activity patterns of patients with diabetic foot ulceration: patients with active ulceration may not adhere to a standard pressure off-loading regimen. Diabetes Care. 2003;26(9):2595-2597.

13. Awan M, Proudlock FA, Gottlob I. A randomized controlled trial of unilateral strabismic and mixed amblyopia using occlusion dose monitors to record compliance. Invest. Ophthalmol Vis Sci. 2005;46(4):1435-1439.

14. Dunbar-Jacobs J, Erlen JA, Schlenk EA, et al. Adherence in chronic disease. Ann Rev Nurse Res. 2000;18:48-90.

15. Ramsey DJ, Arden GB. Rods, dark adaption, and the development of diabetic retinopathy. Clin Opthalmol Res. 2015;1:1-12.

16. Robinson J, Bayliss SC, Fielder AR. Transmission of light across the adult and neonatal eyelid in vivo. Vision Res. 1991;31(10):1837-1840.

17. Hamer RD, Nicholas SC, Tranchina D, et al. Toward a unified model of vertebrate rod phototransduction. Vis Neurosci. 2005;22(4):417-436.

18. Mitroff SR, Scholl BJ. Seeing the disappearance of unseen objects Perception. 2004;33(10):1267-1273.

19. Yau JW, Rogers SL, Kawasaki R, et al. Global prevalence and major risk factors of diabetic retinopathy. Diabetes Care. 2012;35(3):556-564. 
20. Robin DM. Variations in patients' adherence to medical recommendations a quantitative review of 50 years research. Med Care. 2002;42(3):200 209.

21. Claxton AJ, Cramer J, Pierce C. A systematic review of the association between dose regimens and medication compliance. Clin Ther. 2001;23(8):1296-1310

22. Kass MA, Meltzer DW, Gordon M, et al. Compliance with topical pilocarpine treatment. Am J Ophthalmol. 1986;101(5):515-523.

23. Fielder AR, Auld R, Irwin M, et al. Compliance monitoring in amblyopia therapy. Lancet. 1994;343(8896):547.

24. Ohayon MM, Vecchierini MF. Normative sleep data, cognitive function and daily living activities in older adults in the community. Sleep. 2005;28(8):981-989.

25. Lauderdale DS, Knutson KL, Yan LL, et al. Objectively measured sleep characteristics among early- middle - aged adults: the CARDIA study. Am J Epidemiol. 2006;164(1):5-16.
26. Vgntzas AN, Liao D, Pejovic S, et al. Insomnia with objective short sleep duration is associated with type 2 diabetes: a population based study. Diabetes Care. 2009;32(11):1980-1985.

27. Stewart CE, Moseley MJ, Stephens DA, et al. Treatment dose-response in amblyopia therapy: The monitored occlusion treatment of amblyopia study (MOTAS). Invest Ophthalmol Vis Sci. 2004;45(9):3048-3054.

28. Blankenship GW. Diabetic macular edema and argon laser photocoagulation: a prospective randomized study. Ophthalmol. 1979;86(1):69-78.

29. Browning DJ, Fraser CM. The predictive value of patient and eye characteristics on the course of subclinical diabetic macular edema. Am J Ophthalmol. 2008;145(1):149-154. 